\title{
Effect of intravenous steroid added to caudal local anesthetics in improving postoperative pain: A systematic review and meta-analysis
}

\author{
Hiromasa Kawakami, Takahiro Mihara, Nobuhito Nakamura, Koui Ka \\ Kanagawa Children's Medical Center, Kanagawa, Japan
}

\section{Background:}

Caudal anesthesia has been used for postoperative pain control in pediatric surgical patients, but the duration of the analgesic effect is sometimes not satisfactory. The additional caudal medications are investigated but there is some concern regarding safety. Intravenous steroid has been shown effective for pain management after some surgeries.

\section{Method:}

This study was a systematic review and meta-analysis.

A search was conducted of published literature in MEDLINE, EMBASE, Web of Science, and Cochrane Central Register of Control Trials databases.

Randomized control trials that compared intravenous steroid with placebo for pediatric patients who received caudal anesthesia for surgery were included. Continuous data were summarized using mean difference with a 95\% confidence interval $(\mathrm{Cl})$. If the $95 \% \mathrm{Cl}$ included a value of 0 , we considered the difference not to be statistically significant. We used the random effect model (Dersimonian and Laird method) to combine the results. Heterogeneity was quantified with the $I^{2}$ statistic. The primary outcome from the present meta-analysis was analgesic duration.

We contacted the author of an article with missing data and obtained information after the deadline of the abstract.

\section{Result and Discussion:}

Total 998 records were identified through database search and six trials (424 patients) were included in the meta-analysis.

All the studies compared dexamethasone with placebo

Dexamethasone prolonged the analgesic duration with caudal analgesia (mean difference of 244 minutes, 95\% confidence interval 195 to 293). However, this result should be interpreted with caution because of the extreme heterogeneity with $R^{2}$ of $94.8 \%$.

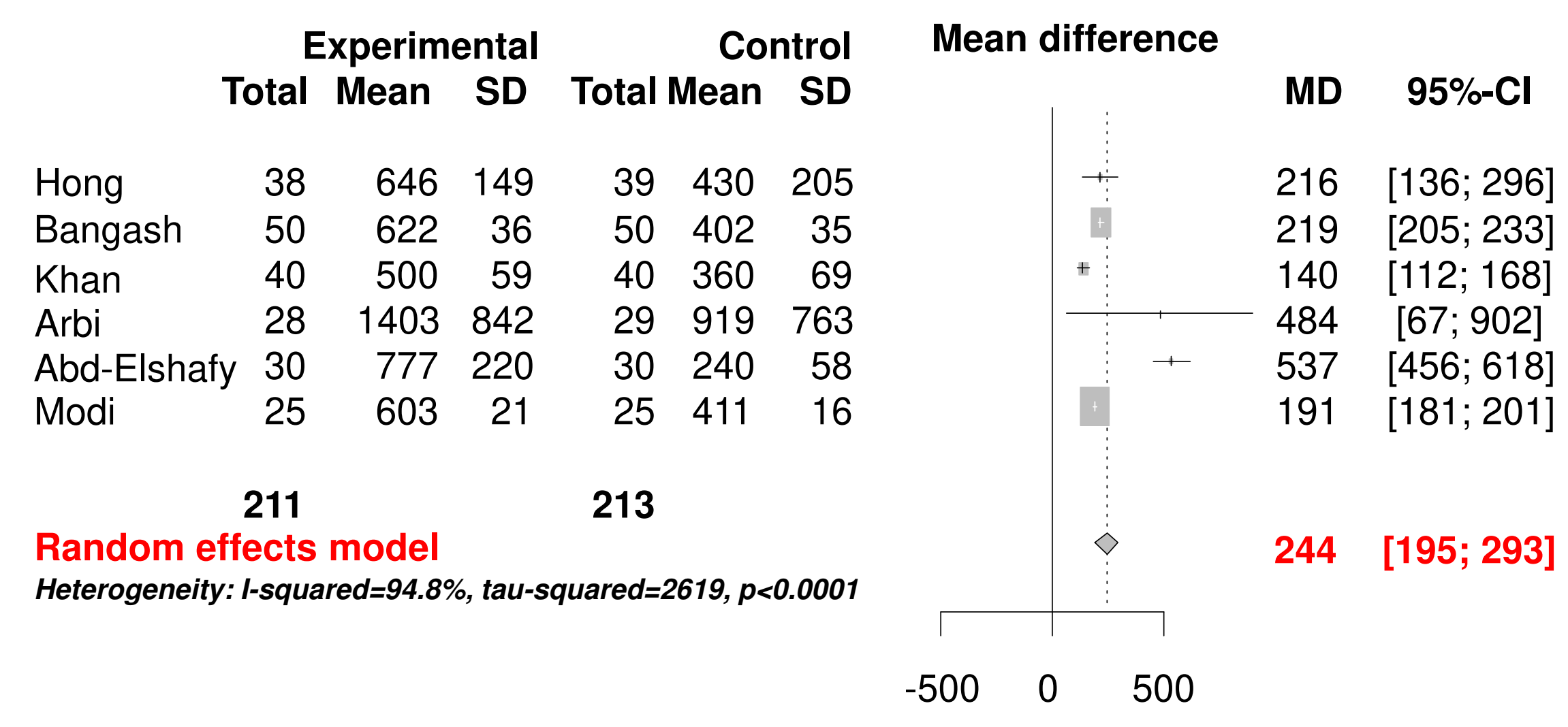

Reference

1. Hong JY et al. Br J Anaesth. 2010;105(4):506-10

2. Bangash $L R$ et al. Biomedica. 2014;30(4):267-71.

3. Khan A et al. Ski J Med Sci. 2015;18(1).

4. Arbi MSA, et al. Middle East $\mathrm{J}$ Anaesthesiol. 2015 Jun;23(2):177-83.

5. Abd-Elshafy SK et al. Pain Physician. 2016;19(6):E841-52.

6. Modi TM et al. Int J Adv Case Reports. 2016;3(11):438-44.

\section{Conclusion:}

